\title{
SEGREGAÇÃO NATURAL DE VIRÓIDES DOS CITROS DETECTADA NA INDEXAÇÃO BIOLÓGICA E BIOQUÍMICA E CONFIRMADA PELOS SINTOMAS DE PLANTAS DE CAMPO ${ }^{1}$
}

\author{
EDUARDO SANCHES STUCHI ${ }^{2}$, LUIZ CARLOS DONADIO ${ }^{3}$, OTÁVIO RICARDO SEMPIONATO ${ }^{4}$
}

\begin{abstract}
RESUMO - Estudaram-se os procedimentos de indexação biológica e bioquímica em um experimento de avaliação do uso de viróides como agentes ananicantes para pomeleiro 'Marsh Seedless'. Em algumas parcelas, houve segregação de viróides, sendo que, em duas, a segregação foi de CEVd (viróide da exocorte dos citros) e, em outras duas, não foi detectada a presença de CVd-II (viróide dos citros II). Como conseqüência desta segregação, não ocorreram sintomas típicos da exocorte em cidra nas parcelas nas quais houve segregação de CEVd, o que ocorreu nas parcelas em que a segregação foi de CVd-II, indicando que é necessária a presença de CEV para a manifestação de sintomas típicos de exocorte em cidra. Numa quinta parcela, não se detectaram viróides, provavelmente, devido à ausência dos mesmos no material de inoculação do teste biológico (escape), já que a planta de campo teve desenvolvimento vegetativo normal e ausência de sintomas no tronco. Finalmente, em uma sexta parcela, o que ocorreu, foi uma segregação drástica, não relatada na literatura, já que a planta de campo mostrou redução de porte e sintomas no tronco. A coleta de varetas para indexação deve ser mais rigorosa em função da ocorrência de segregação.
\end{abstract}

Termos para indexação: cidra, exocorte, Citrus paradisi, pomelo, inoculação, Citrus medica

\section{SEGREGATION OF CITRUS VIROIDS DURING BIOLOGICAL AND BIOCHEMICAL INDEDEXING}

ABSTRACT - The procedures for biological and biochemical indexing were studied in an experiment to evaluate the use of viroids as dwarfing agents for 'Marsh Seedless' grapefruit. In some plots there was viroids segregation. In two plots the segregation was of CEVd (Citrus exocortis viroid) and in other two the presence of CVd-II (Citrus viroid II) was not detected. As a consequence of the segregation, there were no typical citron exocortis symptoms in the portions in which there was segregation of CEVd. Symptoms occurred in the portions, which there was the segregation was of CVd-II, indicating that the presence of CEV is necessary for the manifestation of typical symptoms of exocortis in citron. Viroids were not detected in a fifth plot, probably due to their absence in the inoculation material of the biological test (escapes), since the field plants showed normal vegetative development and absence of symptoms in the trunk. Finally, a drastic segregation, not referred to in the literature, occurred in a sixth plot, since the field plant showed canopy reduction and symptoms in the trunk. As a conclusion budwood sampling for viroid indexing must be more accurate.

Index terms: citron, exocortis, Citrus paradisi, grapefruit, inoculation, Citrus medica

A presença de doença em plantas cítricas pode ser detectada através da indexação ou testes biológicos, procedimento que consiste na união dos tecidos pela enxertia de um hospedeiro suspeito numa planta indicadora sensível; no caso de estas plantas sensíveis inoculadas deixarem de exibir sintomas, tem-se a indicação da ausência do viróide do material testado (Olson, 1968). Atualmente, de forma conjunta, paralela ou alternativa, se faz também a indexação por métodos bioquímicos e de biologia molecular (Roistacher, 1991). As indexações biológicas e bioquímicas são fundamentais para qualquer programa de melhora sanitária e de certificação de citros quando se pensa no estudo da utilização de viróides como agentes ananicantes, visando ao estabelecimento de pomares de alta densidade, isto para se comprovar a contaminação e avaliar a eficiência dos métodos de inoculação (Stuchi, 1993; Stuchi et al., 1998; Roistacher, 1994).

Os viróides dos citros apresentam distribuição irregular. Há relatos, nos quais só se conseguiu detectá-los em um dos oitos setores de uma planta contaminada (Roistacher, 1991); por isso, esse autor recomenda que, para indexação de rotina, pelo menos quatro varetas devem ser coletadas, uma em cada quadrante da planta e, para reindexação de bloco de matrizes, devem ser coletadas oito varetas ao redor da planta. Fernandes (1983), estudando a distribuição do viróide da exocorte em diferentes órgãos da laranjeira-'Baía' (Citrus sinensis L Osbeck) sobre cinco diferentes porta-enxertos, confirmou que o viróide é sistêmico, mas está distribuído em todos os órgãos da planta cítrica de uma maneira irregular. Corrales-Giraldo (1990) afirmou

1 Trabalho $n^{0}$ 077/2000. Recebido: 25/05/2000. Aceito para publicação: 04/06/2001.

2 Parte da dissertação de mestrado do $1^{\circ}$ autor apresentada na FCAV-UNESP, campus de Jaboticabal.

3 Prof. Fruticultura FCAV-UNESP - Campus de Jaboticabal

4 Eng. ${ }^{\circ}$ Agr $^{\circ}$ Estação Experimental de Citricultura de Bebedouro, Caixa Postal, 74, CEP 14700-970 Bebedouro-SP eecb@coopercitrus.com.br 
que: “As estirpes do viróide da exocorte estão distribuídas na parte aérea dos limoeiros de maneira irregular não obedecendo a nenhum tipo de distribuição específica e que, numa planta de limão-'Tahiti' clone 'Quebra-Galho', podem existir áreas com estirpes fortes, severas, moderadas ou fracas do patógeno e mesmo partes completamente livres do viróide. Todavia, os dados sugerem que, na parte Norte-Alta, se encontram as estirpes mais severas, enquanto, na parte Sul-Baixa, estão as mais fracas". Salibe (1961) afirmou ser possível a obtenção de borbulhas livres de exocorte, a partir de árvores contaminadas, através de enxertias sucessivas.

Objetivou-se, com o presente trabalho, estudar a relação entre os resultados da indexação biológica e bioquímica de viróides com o comportamento no campo de plantas inoculadas com diferentes isolados empregados como agentes ananicantes para pomeleiro 'Marsh Seedless' sobre Poncirus trifoliata, durante a qual foi observada uma segregação natural destes patógenos.

Selecionaram-se cinco plantas existentes no Centro de Citricultura "Sylvio Moreira" em função dos sintomas de exocorte que apresentavam. Cada uma dessas plantas constituiu-se num isolado. Esses isolados apresentavam as seguintes características: Isolado 1 - Origem: laranjeira-'Hamlin' (clone velho) enxertada em citrangeiro 'Troyer', do ensaio de comparação entre clones velhos e clones novos (nucelares), plantado em 1955; Isolado 2 - Origem: laranjeira-'Baianinha' (clone velho) enxertada em limoeiro-'Cravo', do ensaio de comparação entre clones velhos e clones novos (nucelares), plantado em 1955; Isolado 3 - Origem: laranjeira-'Hamlin' (clone velho) enxertada em laranjeira-'Caipira', tratamento 7 (exocorte forte) do ensaio de melhoramento de laranjeira-'Hamlin', plantado em 1962; Isolado 4 - Origem: laranjeira-'Hamlin' (clone velho) enxertada em laranjeira-'Caipira', tratamento 5 (exocorte fraca) do ensaio de melhoramento de laranja-'Hamlin', plantado em 1962; Isolado 5 - Origem: laranjeira-'Hamlin' (clone velho) enxertada em limoeiro- 'Cravo' com características de nanismo nas árvores de campo sem apresentar sintomas clássicos da exocorte, do ensaio de comparação entre clones velhos e clones novos (nucelares), plantado em 1982.

Anteriormente, mudas sadias de pomeleiro 'Marsh Seedless'(Citrus paradisi Macf.) enxertadas sobre Poncirus trifoliata foram plantadas no campo, em janeiro de 1991, com espaçamento de $7 \mathrm{~m}$ entre linhas e $3 \mathrm{~m}$ entre plantas e conduzidas de acordo com os tratos culturais habituais e sem irrigação. A inoculação dos diferentes isolados, nas plantas de pomeleiro a campo, foi realizada no campo, seis meses após o plantio (julho de 1991), através de enxertia de duas borbulhas em cada planta, sendo uma no tronco da copa e outra no tronco do cavalo; após o pegamento, as gemas tiveram seu desenvolvimento impedido pela retirada dos brotos. Os tratamentos do experimento foram os seguintes: inoculação dos isolados 1 a 5 (tratamentos 1 a 5), e uma testemunha, o tratamento 6. Cada parcela era constituída de duas plantas inoculadas com o mesmo isolado, exceto o tratamento-testemunha que não recebeu inoculação. Foram empregadas cinco repetições com delineamento em blocos casualizados.

Realizou-se a indexação de todas as plantas do experimento, utilizando-se, como plantas indicadoras, mudas de cidreira 'Etrog 861-S-1 enxertada sobre limoeiro- 'Cravo' em 0501-1995. Foram coletadas varetas de cada árvore de campo de pomeleiro, seguindo-se o esquema de casualização proposto por Greve et al. (1991). Para cada árvore de campo inoculada com cada uma das fontes, em julho de 1991, havia uma planta de cidreira correspondente na casa de vegetação, como planta indicadora. Paralelamente, as árvores de pomeleiro sobre P.trifoliata (inóculo) foram examinadas para a constatação da presença de sintomas da exocorte no tronco do porta-enxerto e tiveram suas alturas e diâmetros medidos para avaliar o grau de redução no seu tamanho. Repetiu-se, para a indexação, o delineamento do experimento de campo, com as parcelas em casa de vegetação (cidreira), recebendo a mesma denominação das parcelas de campo (pomeleiro). A leitura deu-se cinco meses após a inoculação, quando foram atribuídas notas para os sintomas de exocorte em cidreira. As notas foram atribuídas conforme a seguinte escala: $0=$ sem sintomas; $1=$ sintoma fraco; $2=$ sintoma intermediário; 3 = sintoma forte. Estes procedimentos se basearam nas recomendações de Müller \& Costa (1991). Posteriormente, foram coletados $10 \mathrm{~g}$ de ápices de cada uma das 60 plantas de cidreira empregadas no teste. Estas amostras foram liofilizadas e enviadas ao Instituto Valenciano de Investigaciones Agrarias, Valência (IVIA), Espanha, onde se procedeu à caracterização bioquímica (s-PAGE) de cada um dos isolados, segundo a metodologia descrita por Duran-Vila (1986).

A virulência dos isolados em cidra Etrog $861 \mathrm{~S} 1$, após a inoculação com os diferentes isolados, apresentou diferenças significativas (Tabela 1). Os isolados 1; 2 e 3 apresentaram sintomas em cidra entre intermediários e fortes, enquanto os isolados 4 e 5 e a testemunha não mostraram sintomas. Os resultados expressos na Tabela 1 , referentes à classificação dos sintomas em cidra, indicaram a presença de CEV (CEV- citrus exocortis viroid) nos isolados $1 ; 2$ e 3 , bem como a ocorrência de um viróide, ou uma mescla de viróides distintos de $\mathrm{CEV}$, no isolado 4, enquanto o isolado 5 e a testemunha estariam livres de viróides.

Os resultados das análises dos viróides presentes nos isolados por sPAGE, expressos na Tabela 1, confirmam a indicação ao mostrarem a presença do viróide da exocorte dos citros nos isolados 1; 2 e 3, associado a outros dois viróides, CV-II e CVIII. O isolado 4 não contém CEV, mas possui CV- II e CV-III. Já o isolado 5 está livre de viróides. Os isolados 1 a 4 causaram redução no porte das plantas (Tabela 1). Essa redução foi , aproximadamente, de 25;21; 4 e 18\% na altura das plantas, para os isolados 1; 2; 3 e 4, respectivamente. Para o diâmetro da copa, a redução foi de 16;17; 22 e 16\%, para os isolados $1 ; 2 ; 3$ e 4, respectivamente. Os resultados obtidos mostram claramente que a inoculação feita no campo, aos seis meses do plantio, com uma borbulha no porta-enxerto e outra na copa, é suficiente para se conseguir redução significativa no desenvolvimento vegetativo.

Os resultados das análises do conteúdo em viróides das diversas fontes por sPAGE, expressos na Tabela 2, confirmam a indicação ao mostrarem a presença do viróide da exocorte dos citros (CEV- citrus exocortis viroid) nas fontes 1; 2 e 3, associado a outros dois viróides, $\mathrm{CV}$-II e CV- III. A fonte 4 não contém $\mathrm{CEV}$, mas possui CV- II e CV-III. Já a fonte 5 está livre de viróides. A fonte 4, apesar de conter [CV-II + CV-III], não mostrou sintomas de anelamento do pecíolo e epinastia fraca, devida ao anelamento do pecíolo, conforme descrito por Duran-Vila et al. (1993). Tal fato se deve a que cidras inoculadas por viróides dos grupos $\mathrm{CV}$ - II e CV - III só desenvolvem sintomas foliares após a realização da segunda desfolha e transferência para câmara de crescimento (Rodrigues et al., 1999). 
Nas parcelas 1 D1, 2 B, 1 E, e 2 A, houve segregação de viróides, sendo que, nas duas primeiras, $\mathrm{o} C \mathrm{CEV}$ não está presente $\mathrm{e}$, nas duas últimas, não foi detectada a presença de CV-II. Hadas et al. (1989) relataram a ocorrência de fenômeno semelhante quando a fonte de viróides usada nos testes biológicos foi pomeleiro 'Marsh Seedless'. Como conseqüência desta segregação, não ocorreram sintomas em cidra nas parcelas $1 \mathrm{D} 1$ e $2 \mathrm{~B}$, o que ocorreu nas parcelas $1 \mathrm{E}$ e $2 \mathrm{~A}$, indicando que é necessária a presença de $\mathrm{CEV}$ para a manifestação de sintomas típicos de exocorte em cidra.

Na parcela 2 D2 de cidreira, não se detectaram viróides, provavelmente, devido à ausência dos mesmos no material de inoculação do teste biológico (escape), já que a planta de pomeleiro inoculada no campo, que forneceu o inóculo para a indexação, teve desenvolvimento vegetativo normal e ausência de sintomas no tronco.

Quanto à parcela $3 \mathrm{C} 2$ de cidreira, o que ocorreu foi uma segregação drástica, não relatada na literatura consultada , já que a planta de campo inoculada e fornecedora de inóculo mostrou redução de porte semelhante às plantas inoculadas com $\mathrm{CEV}$ e sintomas no tronco típicos de exocorte. Em todos os casos, as varetas para indexação foram coletadas de diferentes setores das plantas e casualizadas de acordo com o proposto por Greve et al. (1991), que propoem, inclusive, coleta de um número maior de varetas, quando comparado com as recomendações de Roistacher (1991).

O isolado 4, por causar redução no porte das plantas, sem a ocorrência de sintomas expressivos no tronco e com maior enfolhamento, deve ser o empregado em estudos de redução do porte das plantas cítricas.

TABELA 1 - Virulência dos isolados de exocorte e viróides presentes em cada isolado analisado por sPAGE, e altura, diâmetro da copa de pomeleiro 'Marsh Seedless', 42 meses após a inoculação. Bebedouro, 1995.

\begin{tabular}{|c|c|c|c|c|}
\hline $\begin{array}{l}\text { Tratamentos } \\
\text { (isolados) }\end{array}$ & $\begin{array}{c}\text { M édias } \\
\text { das Notas }{ }^{1}\end{array}$ & Viróides presentes nos isolados & A ltura & D iâm etro \\
\hline 1 & 2,69 & $\mathrm{CEV}+\mathrm{CV}-\mathrm{II}+\mathrm{CV}-\mathrm{III}$ & $2,02 \mathrm{~b}^{2}$ & $2,56 \mathrm{~b}$ \\
\hline 2 & 2,69 & $\mathrm{CEV}+\mathrm{CV}-\mathrm{II}+\mathrm{CV}-\mathrm{III}$ & $2,14 \mathrm{~b}$ & $2,52 \mathrm{~b}$ \\
\hline 3 & 1,96 & $\mathrm{CEV}+\mathrm{CV}-\mathrm{II}+\mathrm{CV}-\mathrm{III}$ & $2,07 \mathrm{~b}$ & $2,39 \mathrm{~b}$ \\
\hline 4 & 0 & $\mathrm{CV}-\mathrm{II}+\mathrm{CV}-\mathrm{III}$ & $2,22 \mathrm{~b}$ & $2,55 \mathrm{~b}$ \\
\hline 5 & 0 & nenhum & $2,71 \mathrm{a}$ & 2,99 a \\
\hline 6 & 0 & nenhum & $2,71 \mathrm{a}$ & 3,05 a \\
\hline
\end{tabular}

TABELA 2 - Virulência dos isolados, classificação de sintomas em cidra devido a 5 fontes de exocorte e testemunha sem inocular e ocorrência nas parcelas de viróides determinados por sPAGE.

\begin{tabular}{|c|c|c|c|c|c|c|}
\hline \multirow[t]{2}{*}{ Parcelas } & \multirow{2}{*}{$\begin{array}{c}\text { Sintom as } \\
\text { nas plantas } \\
\text { inóculo }\end{array}$} & \multirow{2}{*}{$\begin{array}{c}\text { Notas }{ }^{1} \text { e } \\
\text { sintom as } \\
\text { em cidreira }\end{array}$} & \multicolumn{3}{|c|}{ Viróides } & \multirow[t]{2}{*}{ O bservação } \\
\hline & & & C E V & $\mathrm{C} \mathrm{V}-\mathrm{II}$ & C V-III & \\
\hline $1 \mathrm{~A}$ & + & $3 *$ & + & + & + & \\
\hline $1 \mathrm{~B}$ & + & 3 & + & + & + & \\
\hline $1 \mathrm{C}$ & + & 3 & + & + & + & \\
\hline $1 \mathrm{D} 1$ & + & $0 * *$ & - & + & + & s e greg a ção \\
\hline $1 \mathrm{D} 2$ & + & $3 * *$ & + & + & + & \\
\hline $1 \mathrm{E}$ & + & 3 & + & - & + & s e greg a ção \\
\hline $2 \mathrm{~A}$ & + & 3 & + & - & + & segreg a ção \\
\hline $2 \mathrm{~B}$ & + & 0 & - & + & + & s egreg a ção \\
\hline $2 \mathrm{C}$ & + & 3 & + & + & + & \\
\hline 2 D 1 & + & $3 * *$ & + & + & + & \\
\hline 2 D 2 & - & $0 * *$ & - & - & - & escape \\
\hline $2 \mathrm{E}$ & + & 3 & + & + & + & \\
\hline $3 \mathrm{~A}$ & + & 3 & + & + & + & \\
\hline $3 \mathrm{~B}$ & + & 3 & + & + & + & \\
\hline 3 C 1 & + & $3 * *$ & + & + & + & \\
\hline 3 C 2 & + & $0 * *$ & - & - & - & segregação drás1 \\
\hline $3 \mathrm{D}$ & + & 3 & + & + & + & \\
\hline $3 \mathrm{E}$ & + & 3 & + & + & + & \\
\hline $4 \mathrm{~A}$ & - & 0 & - & + & + & \\
\hline $4 \mathrm{~B} 1$ & - & 0 & - & + & + & \\
\hline 4 B 2 & + & 0 & - & + & + & sintom as fracos \\
\hline $4 \mathrm{C}$ & - & 0 & - & + & + & \\
\hline $4 \mathrm{D} 1$ & - & 0 & - & + & + & \\
\hline $4 \mathrm{D} 2$ & + & 0 & - & + & + & sintomas fracos \\
\hline $4 \mathrm{E}$ & - & 0 & - & + & + & \\
\hline $5 \mathrm{~A}$ & - & 0 & - & - & - & \\
\hline $5 \mathrm{~B}$ & - & 0 & - & - & - & \\
\hline $5 \mathrm{C}$ & - & 0 & - & - & - & \\
\hline $5 \mathrm{D}$ & - & 0 & - & - & - & \\
\hline $5 \mathrm{E}$ & - & 0 & - & - & - & \\
\hline $6 \mathrm{~A}$ & - & 0 & - & - & - & \\
\hline $6 \mathrm{~B}$ & - & 0 & - & - & - & \\
\hline $6 \mathrm{C}$ & - & 0 & - & - & - & \\
\hline $6 \mathrm{D}$ & - & 0 & - & - & - & \\
\hline $6 \mathrm{E}$ & - & 0 & - & - & - & \\
\hline
\end{tabular}


1 - É necessária a presença do viróide da exocorte para a manifestação dos sintomas típicos em cidra durante a indexação biológica-padrão no Brasil, mesmo quando ocorre infeção com os viróides dos citros $\mathrm{CV}$-II e CV-III.

2 - Ocorre segregação de viróides durante os procedimentos de indexação atuais, inclusive drástica ou total.

3 - As varetas para uso na indexação devem ser coletadas em quadrantes de acordo com os pontos cardeais e em três diferentes alturas das plantas: a altura do peito (setor mediano), do joelho (setor baixo) e no topo das plantas (setor alto), e, posteriormente, casualizadas conforme o padrão de Greve et al. (1991).

\section{REFERÊNCIAS BIBLIOGRÁFICAS}

CORRALES-GIRALDO, J.A. Distribuição do viróide da exocorte em plantas de limão 'Tahiti', Citrus latifolia Tanaka. 1990. 87f. Dissertação (Mestrado em Agronomia) - Faculdade de Ciências Agronômicas, Universidade Estadual Paulista, Botucatu, 1990.

DURAN-VILA, N.; PINA, J.A.; NAVARRO, L. Improved indexing of citrus viroids. In: CONFERENCE OF THE INTERNATIONAL ORGANIZATION OF CITRUS VIROLOGISTS, 12., 1992. Nova Delhi. Proceedings... Riverside: International Organization of Citrus Virologists, 1993. p.202-211.

FERNANDES, E.A. Distribuição do viróide da exocorte em diferentes órgãos de laranjeiras 'Baianinha' (Citrus sinensis (L.) Osbeck), sobre cinco porta-enxertos.1983. 51 f. Dissertação (Mestrado em Agronomia) - Faculdade de Ciências Agronômicas, Universidade Estadual Paulista, Botucatu, 1983.

GREVE, A.; PRATES, H.S.; MÜLlER, G.W. Produção de borbulhas certificadas de citros no Estado de São Paulo. In: RODRIGUEZ,O; VIÉGAS, F.; POMPEU JÚNIOR, J.; AMARO, A.A. Ed. Citricultura brasileira. 2 ed. Campinas: Fundação Cargill, 1991. v.1, p.302-317.

HADAS, R.; BAR-JOSEPH, M.; SEMANCICK, J.S. Segregation of a viroid complex from a graft-transmissible dwarfing agent source for grapefruit trees. Annals Applied of Biology,
Wellesbourne, n.115, p. 515-20, 1989.

MÜLLER, G.W., COSTA, A.S. Doenças causadas por vírus, viróides e similares em citros. In: RODRIGUEZ, O. (Ed.) Citricultura brasileira. 2. ed. Campinas: Fundação Cargill, 1991. v.2, p.744-751.

OLSON, E.O. Review of recent research on exocortis disease. In: CONFERENCE OF THE INTERNATIONAL ORGANIZATION OF CITRUS VIROLOGISTS, 4. 1966, Roma. Proceedings... Gainesville. University Florida Press, 1968. p. 92-6.

RODRIGUES, M.I.S.; BARBOSA, C.J.; SANTOS FILHO, H.P.; CARVALHO, O.L.S., VILARINHOS, A.D. Uso de desfolha na indução de sintomas em cidra 'Arizona $861 \mathrm{S1}$ ' infectada por viróides dos citros. Revista Brasileira de Fruticultura, Jaboticabal-SP, v.21, n.3, p.338-41, 1999.

ROISTACHER, C.N. Graft-transmissible diseases of citrus: handbook for detection and diagnosis. Rome: IOCV, FAO, 1991. 286p.

ROISTACHER, C.N. Dwarfing of citrus by use of citrus viroids Pros and Cons. In: INTERNATIONAL CITRUS CONGRESS, 7., 1992, Acireale. Proceedings... Acireale: International Society of Citriculture, 1994. P.791-96

SALIBE, A.A. Contribuição ao estudo da doença exocorte dos citros. 1961. 76 f. Tese (Doutorado em Agronomia) - Escola Superior de Agricultura "Luiz de Queiróz", Universidade São Paulo, Piracicaba, 1961.

STUCHI, E.S. El control del tamaño de los arboles en plantaciones comerciales de citricos. 1993.45f. Tesis (Master en Citricultura) - Universidad Politecnica de Valencia, Valencia, 1993.

STUCHI, E.S.; DONADIO, L.C.; SEMPIONATO, O.R.; SILVA, J.A.A. Caracterização de cinco isolados de exocorte e efeitos de sua inoculação no desenvolvimento vegetativo e produção inicial de pomeleiro 'Marsh Seedless' (Citrus paradisi Macf. Revista Brasileira de Fruticultura, Cruz das Almas, v.20, n.2, p.158-66, 1998. 